Published in final edited form as:

Small. 2015 January 7; 11(1): 96-102. doi:10.1002/smll.201402118.

\title{
Selective Breakdown of Metallic Pathways in Double-Walled Carbon Nanotube Networks
}

\author{
Allen L. Ng, \\ Department of Chemistry and Biochemistry, University of Maryland, College Park, MD 20742, \\ United States \\ Dr. Yong Sun, \\ Department of Chemistry and Biochemistry, University of Maryland, College Park, MD 20742, \\ United States \\ Lyndsey Powell, \\ Department of Chemistry and Biochemistry, University of Maryland, College Park, MD 20742, \\ United States \\ Dr. Chuan-Fu Sun, \\ Department of Chemistry and Biochemistry, University of Maryland, College Park, MD 20742, \\ United States

\section{Prof. Chien-Fu Chen,} \\ Graduate Institute of Biomedical Engineering, National Chung Hsing University, Taiwan \\ Prof. Cheng S. Lee, and \\ Department of Chemistry and Biochemistry, University of Maryland, College Park, MD 20742, \\ United States

\section{Prof. YuHuang Wang} \\ Department of Chemistry and Biochemistry, University of Maryland, College Park, MD 20742, \\ United States \\ Chien-Fu Chen: stevechen@nchu.edu.tw; YuHuang Wang: yhw@umd.edu
}

\section{Abstract}

Covalently functionalized, semiconducting double-walled carbon nanotubes exhibit remarkable properties and can outperform their single-walled carbon nanotube counterparts. In order to harness their potential for electronic applications, metallic double-walled carbon nanotubes must be separated from the semiconductors. However, the inner wall is inaccessible to current separation techniques which rely on the surface properties. Here we describe the first approach to address this challenge through electrical breakdown of metallic double-walled carbon nanotubes, both inner and outer walls, within networks of mixed electronic types. The intact semiconductors demonstrate a $\sim 62 \%$ retention of the ON-state conductance in thin film transistors in response to covalent functionalization. The selective elimination of the metallic pathways improves the

\footnotetext{
Correspondence to: Chien-Fu Chen, stevechen@nchu .edu.tw; YuHuang Wang, yhw@umd. edu. 
ON/OFF ratio, by more than 360 times, to as high as 40,700, while simultaneously retaining high ON-state conductance.

\section{Graphical abstract}

Removal of metallic nanotubes from the semiconductors is an essential step to make use of double-walled carbon nanotubes for electronic applications. By electrical breakdown of the metallic pathways, high performance thin film transistors with simultaneous high ON-state conductance and high ON/OFF ratios are achieved.

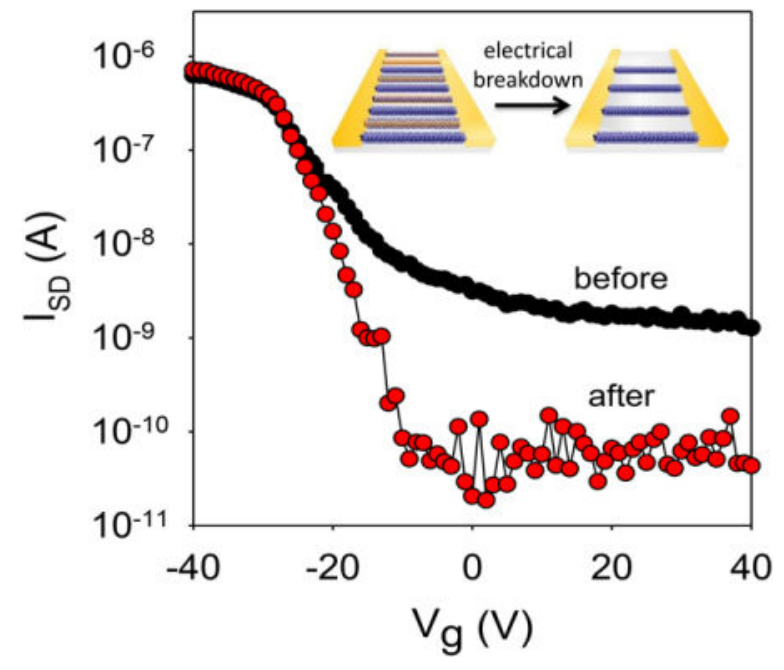

\section{Keywords}

double-walled carbon nanotubes; transistors; electrical breakdown; surface chemistry; sorting

\section{Introduction}

A double-walled carbon nanotube (DWCNT) is comprised of two nested single-walled carbon nanotubes (SWCNTs) that display intricate and interesting electronic properties. ${ }^{[1]}$ This emerging class of carbon nanostructures exhibit unique electrical, ${ }^{[2,3]}$ mechanical, ${ }^{[4,5]}$ and optical properties. ${ }^{[6-8]}$ Recent progress by us ${ }^{[3,9,10]}$ and Martel et al. ${ }^{[11]}$ has further demonstrated that outer wall-selective covalent functionalization of DWCNTs produces a new structure with outer wall protection and SWCNT-like electrical properties stemming from the protected inner tube. Desired surface properties, such as solubility ${ }^{[9]}$ and reactivity of terminal groups for sensing, ${ }^{[2]}$ can be obtained with DWCNTs by judicious selection of functional groups. This ability to tailor surface properties cannot be obtained with SWCNTs due to defect-induced electron scattering, ${ }^{[12]}$ which transforms SWCNTs into insulators even at relatively low degrees of functionalization. ${ }^{[10]}$ Covalent functionalization of DWCNTs provides an unprecedented platform to attach robust functional groups while maintaining the exceptional electronic properties of the inner tube for applications such as high performance sensors with simultaneous sensitivity and selectivity. ${ }^{[2]}$ 
Depending on the nanotube diameter and chiral angle, both the inner and outer walls of a DWCNT can be independently semiconducting or metallic. The semiconducting tubes can have hole mobilities exceeding $70,000 \mathrm{~cm}^{2} \mathrm{~V}^{-1} \mathrm{~s}^{-1}$ while the metallic ones may attain current carrying capacities 1000 times greater than that of copper. ${ }^{[13,14]}$ Due to the unique combination of extremely high carrier mobility, one-dimensional structure, and surface protection, DWCNTs can become ideal building blocks for high performance semiconductor field-effect transistors applications such as logic gates, $\mathrm{p}$-n diodes, and single molecule sensors. ${ }^{[15-19]}$

A major issue that impedes the advance in DWCNT electronics is associated with the structural complexity of DWCNTs. ${ }^{[4,7]}$ A DWCNT possessing a metallic tube in either the inner or outer wall will primarily display metallic electronic properties overall regardless of the other wall. While both semiconducting and metallic DWCNTs are intriguing for a variety of applications, the metallic components within semiconductor devices would inhibit efforts to turn off a transistor and contribute to potential electrical shorting of the circuit. High semiconducting purity is essential for exceptional electronic performance such as high ON-state conductance and ON/OFF ratios. ${ }^{[20,21]}$ The benefits of sorting metallic from semiconducting carbon nanotubes within a mixed CNT array or solution ${ }^{[22,23]}$ have been demonstrated with SWCNTs, for instance, for the development of the first carbon nanotube computer ${ }^{[24]}$ and noncovalently modified electrical sensors. ${ }^{[20,25]}$ Previous studies by Hersam et al., Huh et al., and us have shown the possibility to sort DWCNTs by diameter based on differences in buoyant density, ${ }^{[6,26]}$ chemical reactivity, ${ }^{[27]}$ or even outer wall electronic properties; ${ }^{[28]}$ however, it is not yet possible to eliminate the electrical contribution from metallic inner tubes. Overcoming this challenge is essential in order to harness the potential of DWCNTs for high performance electronics.

First demonstrated by Collins et al., electrical breakdown is a technique used to induce wallby-wall destruction within an individual multi-walled carbon nanotube by applying a high voltage bias resulting in Joule heating. ${ }^{[29]}$ Later studies by Rogers et al. ${ }^{[30,31]}$, Ohno et al. ${ }^{[17]}$, and Maruyama et al. ${ }^{[32]}$ applied the technique towards SWCNT networks to preferentially destroy metallic networks while the semiconducting tubes were turned off. This technique has shown significant promise in obtaining enriched semiconducting SWCNT networks with $99.99 \%$ purity and could be used in the fabrication of CNT computers where extremely high purity is essential. ${ }^{[24]}$

Here we demonstrate that metallic DWCNTs, both inner and outer walls, can be selectively eliminated by electrical breakdown (Figure 1) to simultaneously achieve high ON-state conductance and over two orders of magnitude improvement in ON/OFF ratio within a DWCNT network. Compared with SWCNTs, the challenge of electrical breakdown of DWCNT networks lies in the complex structural composition including small bandgap and large diameter outer wall tubes, ${ }^{[30]}$ low semiconductor composition, and four possible combinations of electronic type stemming from both the inner and outer wall. ${ }^{[3]}$ These factors result in undesired destruction of semiconducting channels during electrical breakdown due to the inability to completely turn off the channel and thermal cross-talk from interconnected metallic channels. By monitoring the electrical breakdown in situ, metallic percolated networks were selectively destroyed using breakdown voltage thresholds 
identified for preserving semiconducting DWCNTs. The intact semiconducting DWCNTs retained both high ON/OFF ratios and high ON-state conductance even after covalent functionalization.

\section{Results and Discussion}

Randomly aligned DWCNT thin film transistors (TFTs) with a DWCNT density of 61 nanotubes per $\mu \mathrm{m}^{2}$ were fabricated using procedures outlined in Figure S1. Using a modified bilayer photolithography technique ${ }^{[33]}$ DWCNT TFTs were fabricated in a three-step procedure involving 1) electrode deposition, 2) fabrication of TFT arrays, and 3) passivation of electrodes using the mask patterns shown in Figure S2. Bilayer photolithography involved the use of an intermediate sacrificial layer of polymethylgluturimide (PMGI) followed by a standard layer of photoresist. The use of this sacrificial layer is particularly important for the complete removal of photoresist from DWCNT TFTs after photolithography steps, which would result in high patterning resolution. The poor resolution arises from the aromatic structure of DWCNT TFTs, which adhere strongly to photoresist via $\pi-\pi$ stacking. Due to its non-aromatic structure, PMGI can be cleanly removed using standard resist remover, such as Remover PG, ensuring good fabrication resolution. ${ }^{[33]}$ Fabrication of TFT arrays involved electrical separation of adjacent transistors using oxygen plasma etching to prevent electrical cross-talk. The channel regions of the electrodes were then passivated with a layer of $\mathrm{SiO}_{2}$ to protect their integrity when exposed to aqueous solutions for procedures such as covalent functionalization.

The performance of the TFTs was evaluated by taking current-voltage $\left(\mathrm{I}_{\mathrm{SD}}-\mathrm{V}_{\mathrm{SD}}\right)$ and transfer characteristic $\left(\mathrm{ISD}_{\mathrm{SD}^{-}} \mathrm{V}_{\mathrm{g}}\right)$ curves to determine the ON-state conductance and the ON/OFF ratio. Before electrical breakdown, the TFTs showed high ON-state conductance (0.5-5.0 $\mu \mathrm{S})$, but low-to-moderate ON/OFF ratios (20-500), which was attributed to significant metallic populations despite the use of outer wall purified DWCNTs to make the networks. The average mobility of the TFTs was $1.5 \mathrm{~cm}^{2} / \mathrm{Vs}$ with a standard deviation of 0.5 $\mathrm{cm}^{2} / \mathrm{Vs}$. This value is comparable with random networks of SWCNTs. ${ }^{[30]}$

When a large source-drain voltage was applied, metallic percolated pathways were selectively heated and destroyed since metallic DWCNTs possess higher electrical non-gated conductance than their semiconducting counterparts. A source-drain voltage of $-100 \mathrm{~V}$ was applied in cycles of 28 seconds until no appreciable gains in ON/OFF ratio were observed. Electrical characterization was performed in situ by monitoring the source-drain current during the breakdown. After each cycle, current-voltage and transfer characteristic curves were taken to determine the electrical performance. Figure 2 shows stepwise decay of the OFF-state conductance at the breakdown voltage while the electrical breakdown cycling was performed. Each abrupt decrease in the source-drain current is indicative of an electrical breakdown event. The cycling was halted upon no additional stepwise losses in conductance. The OFF-state conductance after electrical breakdown was typically in the range of 10-50 pS.

This electrical breakdown procedure produces DWCNT TFTs with ON/OFF ratios as high as 40,700 and concomitant $\mathrm{ON}$-state conductance of approximately $1 \mu \mathrm{S}$ for $15 \mu \mathrm{m}$ (length) 
by $50 \mu \mathrm{m}$ (width) channels. Figure 3 and Table 1 show the transfer curves and tabulated electrical properties for a sample device. The minimum OFF-state conductance for this device $(18 \mathrm{pS})$ can be attributed to thermally excited carriers at room temperature and indicates that the metallic tubes are either completely removed or well below the percolation density. We note that the initial areal density of DWCNTs used here was $61 / \mu \mathrm{m}^{2}$, corresponding to $\sim 44 \mathrm{ng} / \mathrm{cm}^{2}$ as we previously reported. ${ }^{[2]}$ Both the ON/OFF ratio and ONstate conductance of a DWCNT network are dependent on the areal density of nanotubes. ${ }^{[2]}$ As the tube density reduces $20 \%$ (from $44 \mathrm{ng} / \mathrm{cm}^{2}$ to $35 \mathrm{ng} / \mathrm{cm}^{2}$ ), the ON/OFF ratio increases by $\sim 6$ folds (from 600 to 4000 ), but the ON-state conductance decreases to $25 \%$ of the initial value (from $2000 \mathrm{nS}$ to $500 \mathrm{nS}$ ). ${ }^{[2]}$ In stark contrast, by electrical breakdown, we observed simultaneous increase in the ON/OFF ratio, by nearly 80 times (from 522 to 40,700 ), and $\sim 100 \%$ retention of the ON-state conductance (Table 1). Based on this direct comparison, the contribution possible change in tube density during electrical breakdown is rather minor.

During electrical breakdown cycling, we observed three distinctly different regimes with respect to the $\mathrm{ON}$ - and OFF-current (Table 1). The first regime, reproducibly displayed approximately a 2-fold increase in ON-current and mobility and 2-fold decrease in OFFcurrent. These changes can be explained by the removal of contaminants such as amorphous carbon along with metallic percolated pathways within the thin film network, which increase the ON-current and decrease the OFF-current, respectively.

The second regime comprises of abrupt decreases in both ON- and OFF-currents, although the OFF-current decreases over 20 times more than the ON-current. The mobility of TFTs in this regime generally decreases back to initial mobility values of 1.6 to $2.2 \mathrm{~cm}^{2} / \mathrm{Vs}$. This is attributed to the selective destruction of metallic tubes. Moderate decreases of approximately $40 \%$ in ON-current may be due to semiconducting percolation pathways that were destroyed in small quantities from the thermal cross-talk between interconnected metallic and semiconducting percolated pathways within the thin film. These observations are consistent with previous works on electrical breakdown with random SWCNT networks. ${ }^{[30]}$

The third regime is defined by a relatively larger decrease ( $60 \%)$ in ON-current without any appreciable changes in the OFF-current. Significant mobility decrease was observed in this regime with most TFTs having mobilities lower than $1.0 \mathrm{~cm}^{2} / \mathrm{Vs}$. In this regime, there are very few metallic percolated pathways remaining in the conduction channel, as evident by the small (10-50 pS) OFF-state conductance, and semiconducting DWCNTs with large diameter outer walls upon prolonged breakdown voltage become more susceptible to breakdown due to their smaller bandgaps. We note that the regimes are defined based off of the behavior of the ON and OFF state characteristics rather than the number of cycles. Each regime can take multiple cycles although for representative transistors as shown in the text, the first regime typically takes one cycle and the second regime typically takes two.

Compared to SWCNTs, electrical breakdown of DWCNT networks is more challenging due to the complex combination of inner and outer wall types. Furthermore, semiconducting outer walls have smaller bandgaps due to the larger diameters, making it more difficult to differentiate from metallic nanotubes. Both factors can result in unintentional breakdown of 
semiconducting pathways, as evident by the decrease of ON-state conductance (by 40-75\%) within the second and third regimes, due to the inability to completely turn off the channel and prevent thermal cross-talk from interconnected metallic pathways. Additionally, it was observed that as DWCNT densities increase, the selectivity of the breakdown process decreases. Increased packing and interconnections of pathways within the DWCNT network result in more susceptibility of semiconducting pathways to be broken down due to thermal cross talk. The observed difficulties of achieving selective electrical breakdown of dense arrays are consistent with those previous studies of SWCNT networks. ${ }^{[34,35]}$ While the breakdown selectivity gradually diminishes at increased tube density, we observed successful electrical breakdown at DWCNT densities as high as 180 nanotubes $/ \mu \mathrm{m}^{2}$. The ability to eliminate metallic contributions while retaining a high ON-state conductance can be accomplished by cycling up to the second regime.

Unlike SWCNT networks, we found that DWCNT networks do not require gating to achieve the selective breakdown of metallic pathways. No additional selectivity was observed when a gating voltage (as high as $40 \mathrm{~V}$ ) was applied. This non-gated electrical breakdown behavior is similar to that of previous observations with single MWCNTs by Collins et al. ${ }^{[29]}$ We hypothesize that the difference between SWCNTs and DWCNTs originates from lower oxygen doping levels in DWCNTs due to outer wall protection. The lower dopant levels protect the semiconducting DWCNTs from being turned on or reaching the breakdown conductance thresholds even at non-gating conditions. On the other hand, SWCNTs, which lack the outer wall protection found in DWCNTs, can have their entire surface exposed to dopants such as oxygen and can result in substantial conductance at nongating conditions.

Channel length studies show a strong relationship between channel length and breakdown voltage (Figure S3). This channel-length dependence is related to the existence of a power threshold for breakdown. At a fixed voltage, power is inversely related to the resistance and thus the channel length. Longer channel length TFTs have higher resistance and hence require higher breakdown voltages. We noted that channel lengths of $3 \mu \mathrm{m}$ could be broken down completely at a source-drain voltage of $-15 \mathrm{~V}$, while even a source-drain voltage of $-200 \mathrm{~V}$ rarely worked for channel lengths larger than $25 \mu \mathrm{m}$.

To ensure that the electrical breakdown was not destroying semiconducting DWCNTs, DWCNT TFTs that were electrically broken down to the second regime were then covalently functionalized with 4-carboxylbenzene functional groups using diazonium chemistry (Figure 4a), which would remove the contributions of outer walls and all exposed inner tubes (if semiconducting outer walls were damaged by electrical breakdown). ${ }^{[2,10]}$ Covalent functionalization of the DWCNT TFT (Figure 4b,c) was verified using Raman spectroscopy (Figure 4d). Evidence of covalent functionalization includes the disappearance of the outer wall radial breathing mode peak at $100-200 \mathrm{~cm}^{-1}$ and the emergence of the Dband $\left(\sim 1300 \mathrm{~cm}^{-1}\right)$. Together, these spectroscopic features support formation of $\mathrm{sp}^{3}$ defects on the outer wall. Retention of ON-state conductance (Figure 4c) and the preservation of the inner wall RBM peak (Figure 4d) are jointly strong evidence that the inner walls of semiconducting DWCNTs were protected by the outer walls during the covalent modification. This observation is consistent with our previous results that the 
functionalization of a DWCNT network of mixed electronic types leads to a decrease in conductance associated with the loss of outer wall electrical properties, but retains the inner wall conductivity. ${ }^{[10]}$ This experiment suggests that the semiconducting DWCNTs within the network, both inner and outer walls, are immune from electrical breakdown through the second regime.

To further understand the structural features of the DWCNT networks during the three regimes of electrical breakdown, covalent functionalization was used as a probe for intact DWCNTs (Figure 5). Within the first and second regime both the inner and outer walls from semiconducting DWCNTs are intact, as indicated by the retention of ON-state conductance after functionalization (Figure 5b,e). In contrast, the third regime shows minimal retention of $\mathrm{ON}$-state conductance upon functionalization indicative of damaged inner or outer walls, or both within semiconducting percolated pathways (Figure $5 \mathrm{c}, \mathrm{g}$ ), which corroborates the decrease of the ON/OFF ratio in the third regime.

\section{Conclusion}

We demonstrate high performance covalently functionalized DWCNT TFTs through controlled electrical breakdown of metallic percolated pathways without damaging the inner or outer wall of semiconducting DWCNTs. The selective destruction of metallic percolated pathways significantly increased the ON/OFF ratio, by 360 -fold. ON/OFF ratios as high as 40,700 and an ON-state conductance of $600-1000 \mathrm{nS}$ were simultaneously attained. These features are representative of 30 electrically broken down DWCNT TFTs. This combination of high ON/OFF ratio and ON-state conductance in DWCNT networks provides an opportunity to create highly stable, robust thin film devices with tailored surface chemistry and excellent electrical properties.

\section{Experimental Section}

\section{Device fabrication and bilayer photolithography}

Highly n-doped silicon wafers with a $300 \mathrm{~nm}$ thermally grown silicon oxide layer (Silicon Quest International) were used as the back-gated substrates and the gate dielectric respectively. Substrate and DWCNT thin film preparation were prepared using a procedure from previous work. ${ }^{[2]}$ DWCNTs (Unidym DW411UA) possessed average inner and outer wall diameters of 0.86 and $1.61 \mathrm{~nm}$, respectively and an average length of $900 \mathrm{~nm}$. Statistically, semiconducting DWCNTs constitute approximately $4 / 9$ of the entire population, in both mass and numbers. A randomly aligned thin film network with a DWCNT density of approximately 61 nanotubes per $\mu \mathrm{m}^{2}$ was transferred to the substrates. Using bilayer photolithography with PMGI and Shipley 1813 as the sacrificial layer and photoresist respectively, chromium and gold source/drain electrode contacts with a channel length and width of 15 and $50 \mu \mathrm{m}$, respectively were patterned. Using a similar patterning procedure with the mask design shown on Figure S2b, bilayer photolithography was again used to fabricate TFT arrays by separating adjacent transistors from the thin film structure through using oxygen plasma etching. The TFT was patterned so that the DWCNTs confined within the source-drain electrodes were protected with bilayer photoresist while the remaining area was exposed. Exposure of the patterned substrates to oxygen plasma at a 
power of $100 \mathrm{~W}$ and a flow rate of $50 \mathrm{sccm}$ for 30 seconds resulted in the complete removal of all DWCNT networks exposed while the DWCNTs within the protected regions remained intact. The electrodes were then passivated by patterning a $125 \mathrm{~nm}$ layer of $\mathrm{SiO}_{2}$ using bilayer photolithography on top of the inner regions of the electrodes using the mask design shown on Figure S2c.

\section{Characterization}

DWCNT percolated networks confined within the source-drain regions were characterized using scanning electron microscope (SEM) for tube densities and fabrication quality.

\section{Electrical Breakdown and Measurements}

For the electrical breakdown of DWCNT TFTs, a large negative $\mathrm{V}_{\mathrm{SD}}$ bias of $-100 \mathrm{~V}$ was applied to DWCNT TFTs with a conduction channel length of $15 \mu \mathrm{m}$ for 28 seconds as one cycle. Electrical characterization of I-V and transfer curves (Keithley 4200-SCS) was performed after every cycle.

\section{Covalently functionalized networks}

To verify that the DWCNT TFTs remained intact after electrical breakdown, electrically broken down TFTs were functionalized with 4-carboxylbenzenediazonium tetrafluoroborate. The reagent was synthesized from 4-aminobenzoic acid (Sigma Aldrich, 잉) using a previously described method. ${ }^{[10]}$ The diazonium structure was confirmed by ${ }^{1} \mathrm{H}-\mathrm{NMR}$ (Bruker DRX-400) and FTIR (Thermo Nicolet NEXUS 670 with ATR attachment) spectroscopies. For NMR analysis, samples were dissolved in acetonitrile-D3 (99.8\%, Cambridge Isotope Laboratories, Inc). Covalent functionalization was achieved by flowing a $1 \mathrm{mM}$ aqueous solution of 4-carboxylbenzenediazonium tetrafluoroborate at a rate of 25 $\mu \mathrm{L} / \mathrm{min}$ through microfluidic channels attached to the TFTs for one hour. The residual reactants were then removed by flowing Nanopure ${ }^{\mathrm{TM}}$ water through the microfluidic channels for $30 \mathrm{~min}$ at the same flow rate. Electrical characterization of the DWCNT TFTs was performed after functionalization and Raman spectroscopy (Horiba Jobin Yvon LabRAM Raman microscope, model ARAMIS) with an excitation line of $633 \mathrm{~nm}$ was used to characterize the extent of covalent functionalization.

\section{Supplementary Material}

Refer to Web version on PubMed Central for supplementary material.

\section{Acknowledgments}

We wish to acknowledge M. C. Hersam and A. Green for assistance with DGU purification. This research was supported in part by NIH grant CA 143177 and GM 103536 (C. Lee), the Ministry of Science and Technology, Taiwan (NSC 101-2113-M-005-001-MY2) (C. F. Chen), the National Science Foundation early career award CHE - 1055514, and the Office of Naval Research grant N000141110465 (Y. Wang).

\section{References}

1. Shen C, Brozena AH, Wang Y. Nanoscale. 2011; 3:503. [PubMed: 21042608] 
2. Huang J, Ng AL, Piao YM, Chen CF, Green AA, Sun CF, Hersam MC, Lee CS, Wang YH. J Am Chem Soc. 2013; 135:2306. [PubMed: 23327103]

3. Liu KH, Wang WL, Xu Z, Bai XD, Wang EG, Yao YG, Zhang J, Liu ZF. J Am Chem Soc. 2009; 131:62. [PubMed: 19093842]

4. Kim YA, Muramatsu H, Hayashi T, Endo M, Terrones M, Dresselhaus MS. Chem Phys Lett. 2004; 398:87.

5. Peng B, Locascio M, Zapol P, Li SY, Mielke SL, Schatz GC, Espinosa HD. Nat Nanotechnol. 2008; 3:626. [PubMed: 18839003]

6. Green AA, Hersam MC. Nat Nanotechnol. 2009; 4:64. [PubMed: 19119285]

7. Villalpando-Paez F, Son H, Nezich D, Hsieh YP, Kong J, Kim YA, Shimamoto D, Muramatsu H, Hayashi T, Endo M, Terrones M, Dresselhaus MS. Nano Lett. 2008; 8:3879. [PubMed: 18937518]

8. Liu KH, Hong XP, Wu MH, Xiao FJ, Wang WL, Bai XD, Ager JW, Aloni S, Zettl A, Wang EG, Wang F. Nat Commun. 2013; 4:6.

9. Brozena AH, Moskowitz J, Shao BY, Deng SL, Liao HW, Gaskell KJ, Wang YH. J Am Chem Soc. 2010; 132:3932. [PubMed: 20178323]

10. Piao YM, Chen CF, Green AA, Kwon H, Hersam MC, Lee CS, Schatz GC, Wang YH. J Phys Chem Lett. 2011; 2:1577.

11. Bouilly D, Cabana J, Meunier F, Desjardins-Carriere M, Lapointe F, Gagnon P, Larouche FL, Adam E, Paillet M, Martel R. ACS Nano. 2011; 5:4927. [PubMed: 21595426]

12. Goldsmith BR, Coroneus JG, Khalap VR, Kane AA, Weiss GA, Collins PG. Science. 2007; 315:77. [PubMed: 17204645]

13. Durkop T, Getty SA, Cobas E, Fuhrer MS. Nano Lett. 2004; 4:35.

14. Wei BQ, Vajtai R, Ajayan PM. Appl Phys Lett. 2001; 79:1172.

15. Cao Q, Kim HS, Pimparkar N, Kulkarni JP, Wang CJ, Shim M, Roy K, Alam MA, Rogers JA. Nature. 2008; 454:495. [PubMed: 18650920]

16. Javey A, Wang Q, Ural A, Li YM, Dai HJ. Nano Lett. 2002; 2:929.

17. Sun DM, Timmermans MY, Tian Y, Nasibulin AG, Kauppinen EI, Kishimoto S, Mizutani T, Ohno Y. Nat Nanotechnol. 2011; 6:156. [PubMed: 21297625]

18. Kong J, Franklin NR, Zhou CW, Chapline MG, Peng S, Cho KJ, Dai HJ. Science. 2000; 287:622. [PubMed: 10649989]

19. Choi YK, Moody IS, Sims PC, Hunt SR, Corso BL, Perez I, Weiss GA, Collins PG. Science. 2012; 335:319. [PubMed: 22267809]

20. Zhang GY, Qi PF, Wang XR, Lu YR, Li XL, Tu R, Bangsaruntip S, Mann D, Zhang L, Dai HJ. Science. 2006; 314:974. [PubMed: 17095698]

21. Ding L, Tselev A, Wang JY, Yuan DN, Chu HB, McNicholas TP, Li Y, Liu J. Nano Lett. 2009; 9:800. [PubMed: 19159186]

22. Liu HP, Nishide D, Tanaka T, Kataura H. Nat Comm. 2011; 2:8.

23. Wu ZC, Chen ZH, Du X, Logan JM, Sippel J, Nikolou M, Kamaras K, Reynolds JR, Tanner DB, Hebard AF, Rinzler AG. Science. 2004; 305:1273. [PubMed: 15333836]

24. Shulaker MM, Hills G, Patil N, Wei H, Chen HY, Wong P, Mitra S. Nature. 2013; 501:526. [PubMed: 24067711]

25. Chen RJ, Bangsaruntip S, Drouvalakis KA, Kam NWS, Shim M, Li YM, Kim W, Utz PJ, Dai HJ. Proc Natl Acad Sci U S A. 2003; 100:4984. [PubMed: 12697899]

26. Huh JY, Walker ARF, Ro HW, Obrzut J, Mansfield E, Geiss R, Fagan JA. J Phys Chem C. 2010; 114:11343.

27. Deng SL, Piao YM, Brozena AH, Wang YH. J Mater Chem. 2011; 21:18568.

28. Green AA, Hersam MC. ACS Nano. 2011; 5:1459. [PubMed: 21280609]

29. Collins PC, Arnold MS, Avouris P. Science. 2001; 292:706. [PubMed: 11326094]

30. Zhou YX, Gaur A, Hur SH, Kocabas C, Meitl MA, Shim M, Rogers JA. Nano Lett. 2004; 4:2031.

31. Kang SJ, Kocabas C, Ozel T, Shim M, Pimparkar N, Alam MA, Rotkin SV, Rogers JA. Nat Nanotechnol. 2007; 2:230. [PubMed: 18654268]

32. Otsuka K, Inoue T, Chiashi S, Maruyama S. Nanoscale. 2014; 6:8831. [PubMed: 24956406] 
33. Khamis SM, Jones RA, Johnson ATC. AIP Adv. 2011; 1:5.

34. Behnam A, Sangwan VK, Zhong XY, Lian FF, Estrada D, Jariwala D, Hoag AJ, Lauhon LJ, Marks TJ, Hersam MC, Pop E. ACS Nano. 2013; 7:482. [PubMed: 23259715]

35. Shekhar S, Erementchouk M, Leuenberger MN, Khondaker SI. Appl Phys Lett. 2011; 98:243121. 


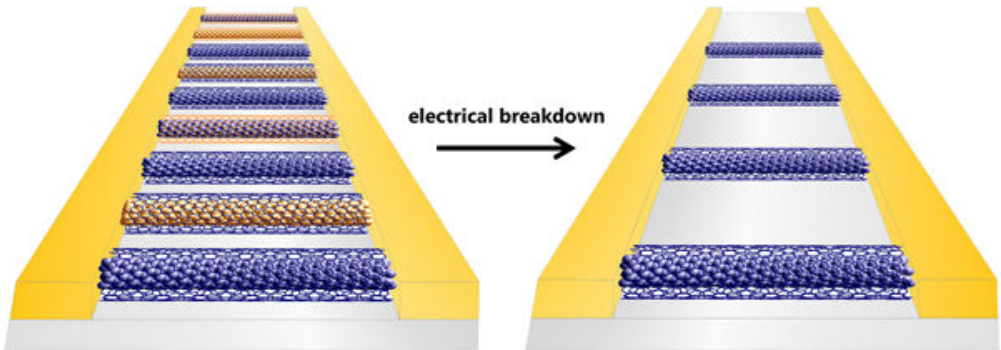

Figure 1.

Schematic of electrical breakdown of double-walled carbon nanotubes. Blue cylinders represent semiconducting tube chiralities, and golden cylinders represent metallic tube chiralities. The four electronic combinations of inner and outer walls are reduced to the semiconducting structure, which, statistically, constitutes $4 / 9$ of the entire population. 
a)

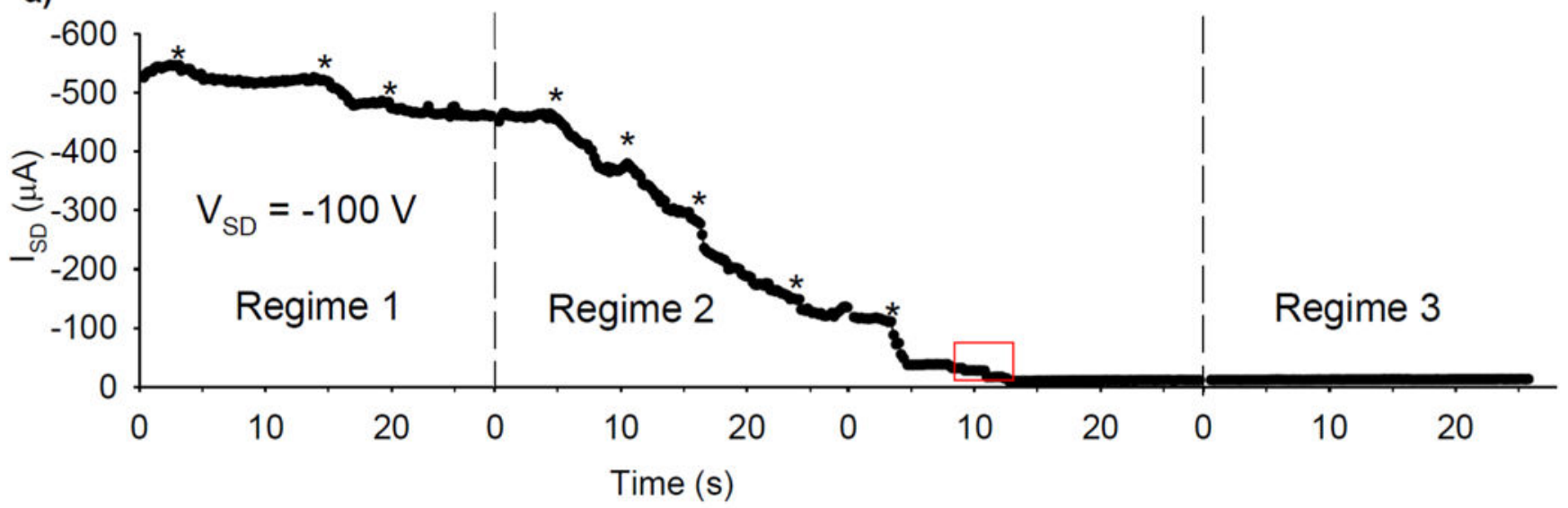

b)
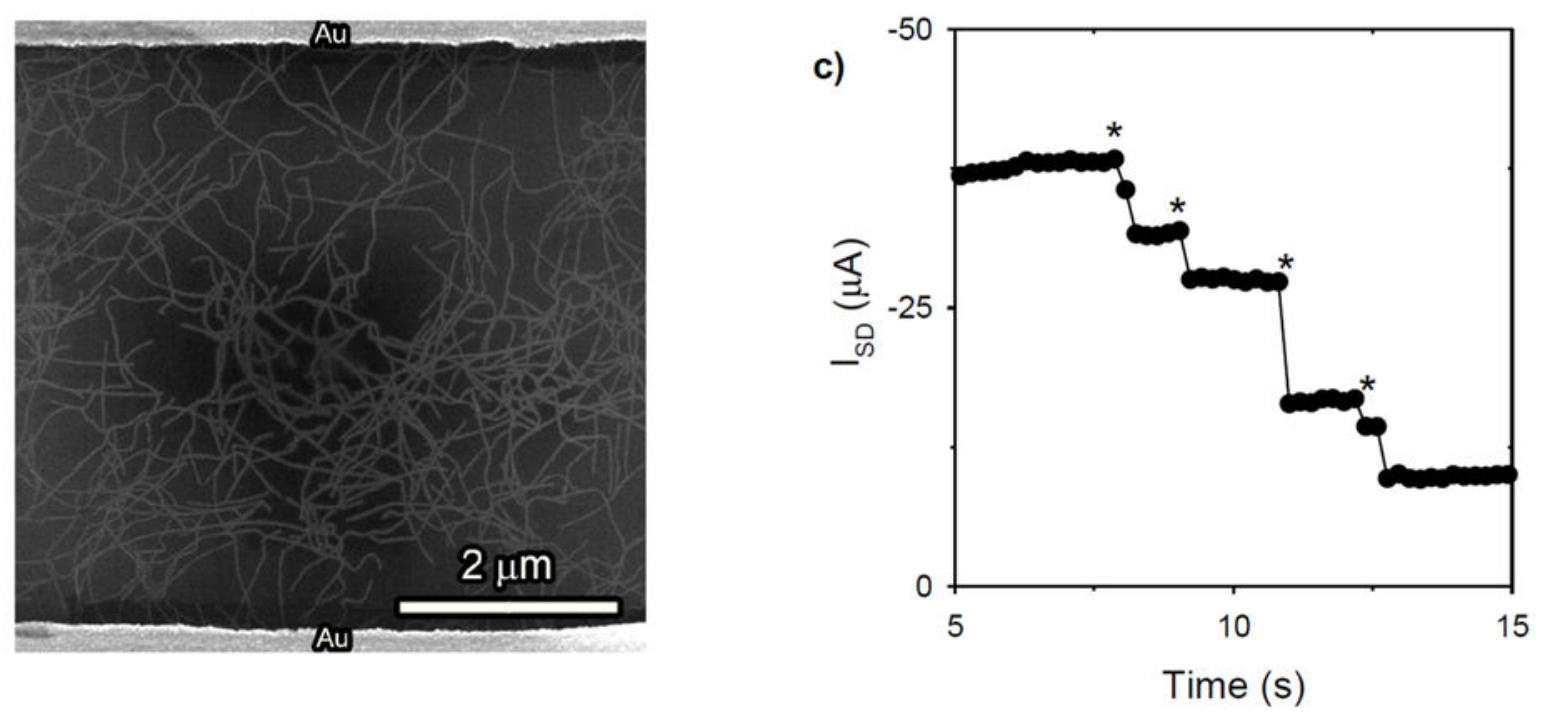

Figure 2.

(a) In situ current versus time measurement during electrical breakdown at a fixed sourcedrain voltage $\left(\mathrm{V}_{\mathrm{SD}}=-100 \mathrm{~V}\right)$ for the three regimes. Electrical breakdown events are characterized by sharp decreases in source-drain current (marked by asterisks). (b) Percolated DWCNT networks characterized by SEM before electrical breakdown. (c) Zoomin of the boxed region in figure (a). 

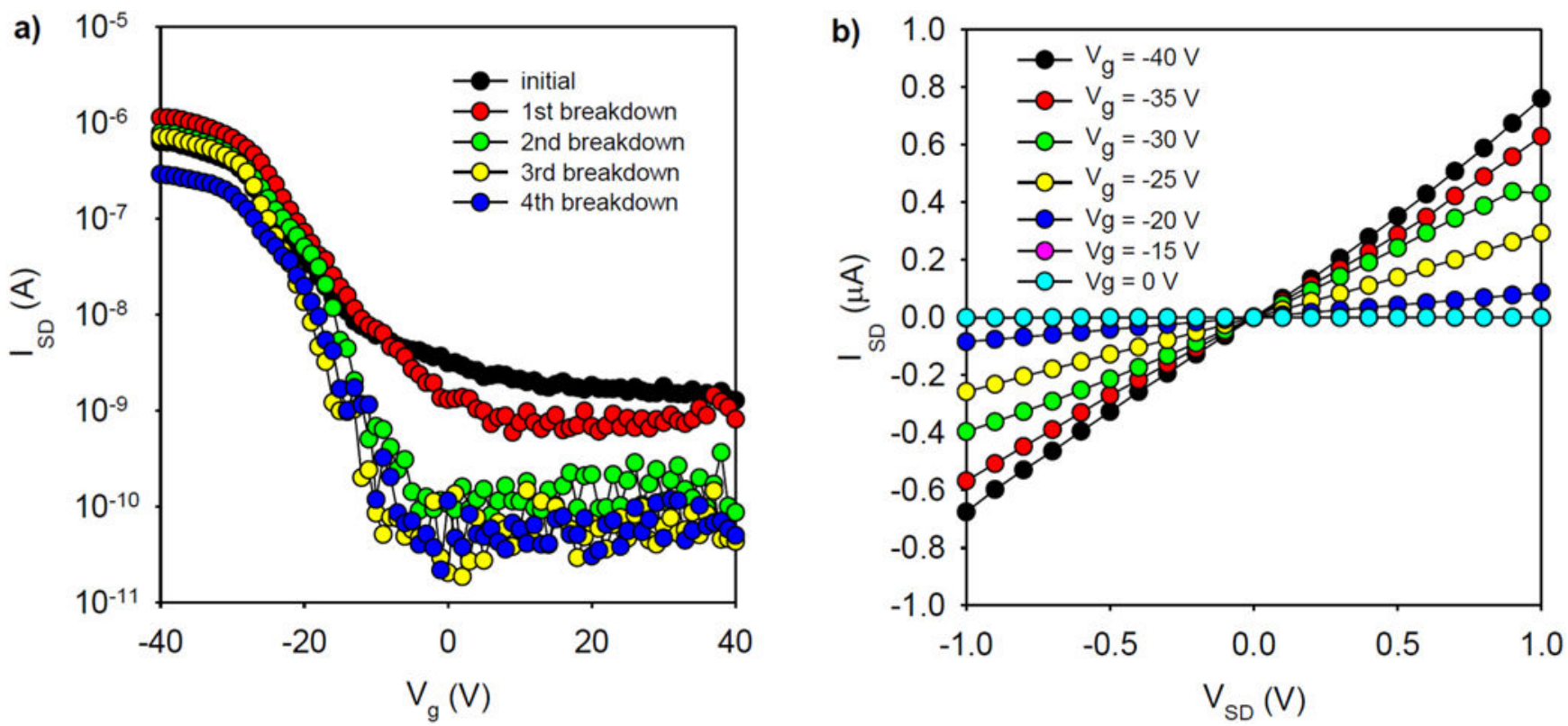

Figure 3.

(a) Transfer properties of a DWCNT TFT after each electrical breakdown event at a fixed source-drain voltage bias $\left(\mathrm{V}_{\mathrm{SD}}=1 \mathrm{~V}\right)$ and $(\mathrm{b}) \mathrm{I}-\mathrm{V}$ curves after the third cycle of electrical breakdown. 
a)

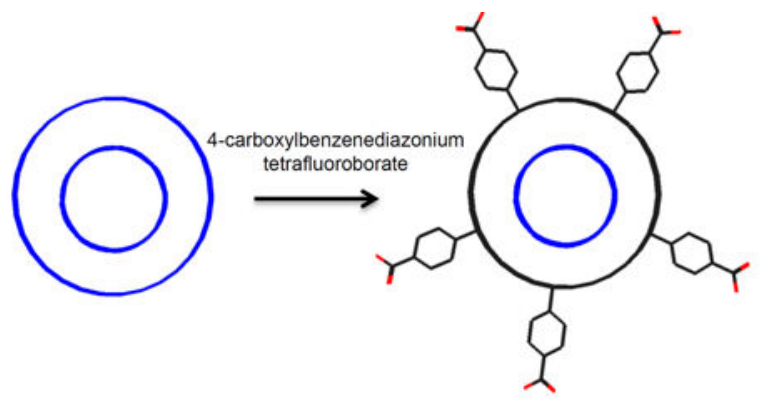

b)
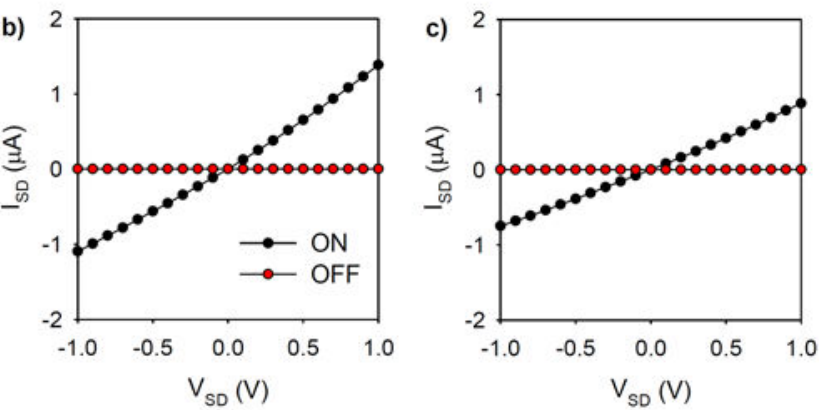

d)

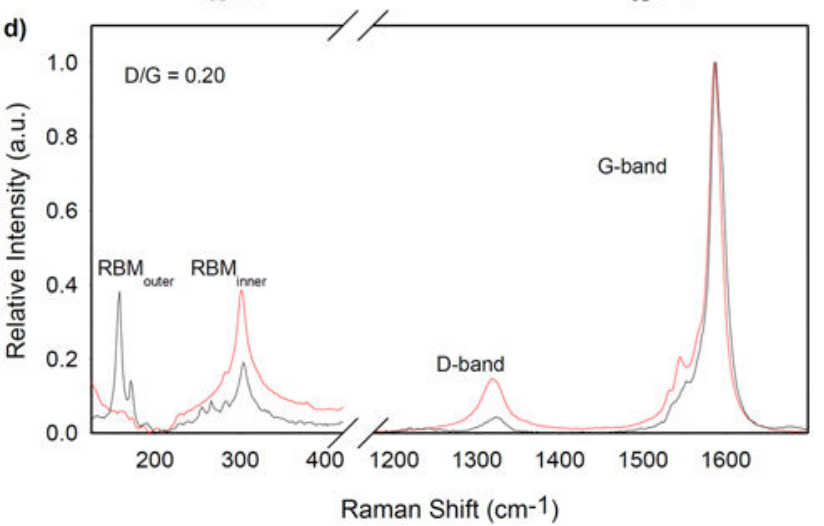

Figure 4.

(a) Outer-wall selective covalent functionalization of a DWCNT with 4-carboxylbenzene groups. Outer-wall contributions are removed upon functionalization. (b) $\mathrm{ON}-\left(\mathrm{V}_{\mathrm{g}}=-40 \mathrm{~V}\right.$, black) and OFF-state $\left(\mathrm{V}_{\mathrm{g}}=40 \mathrm{~V}\right.$, red) I-V curves of a DWCNT TFT within the second regime of electrical breakdown and (c) after outer wall-selective covalent functionalization. (d) Raman spectra of the DWCNT TFT before (black) and after (red) covalent functionalization normalized to G-band intensity. 
a)

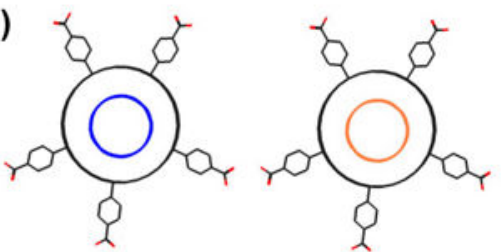

)

b)

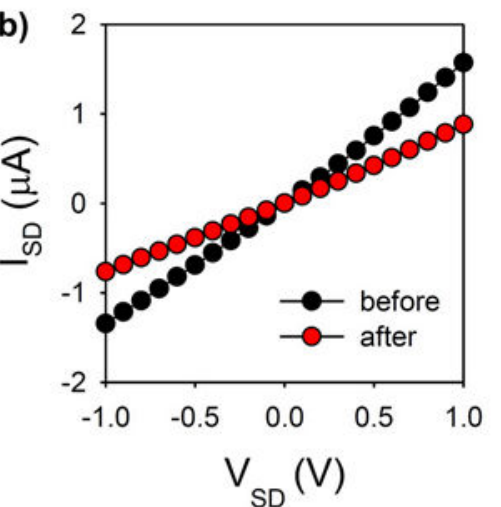

c)

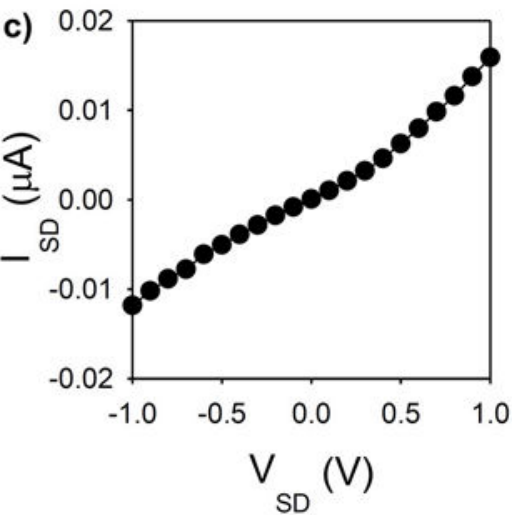

d)

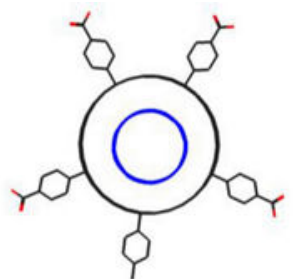

e)

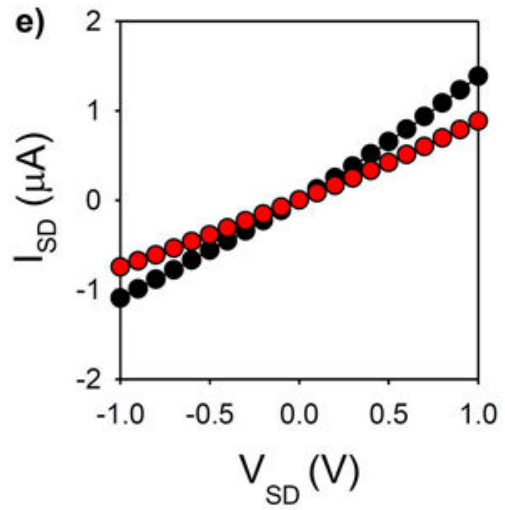

f)

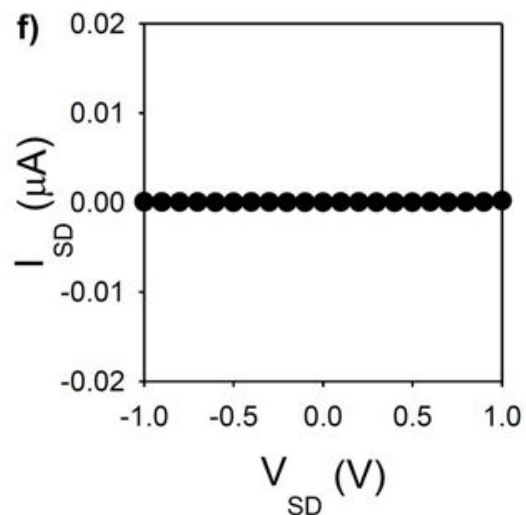

g)
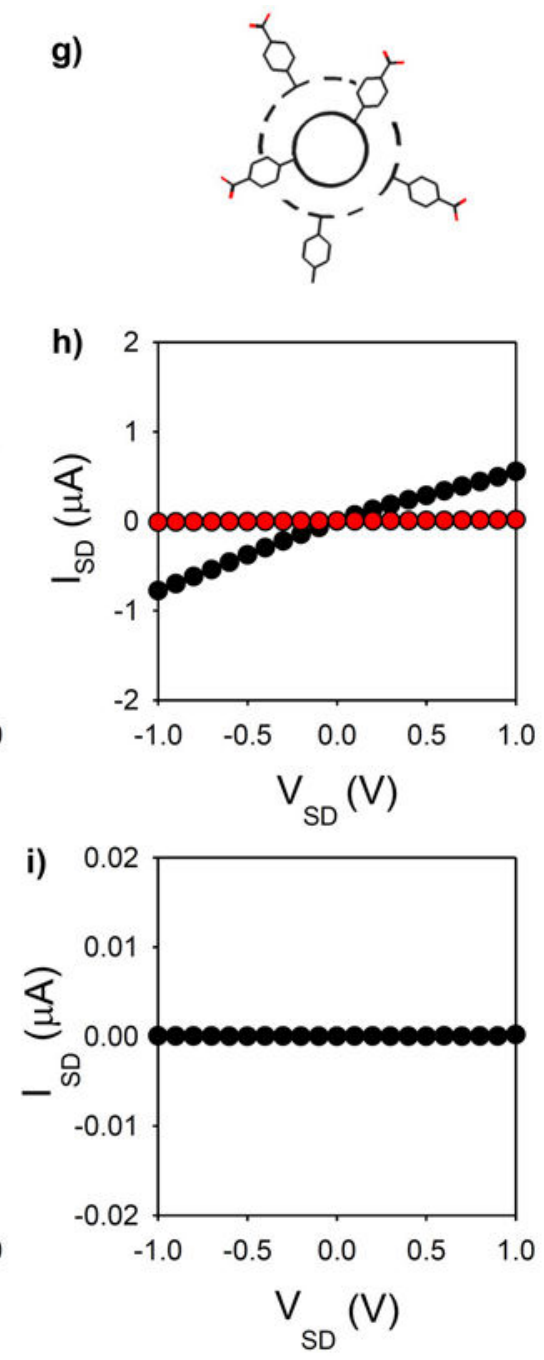

Figure 5.

Outer wall selective chemistry as a probe for intact DWCNT networks for (a) first, (d) second, and (g) third regimes. Blue, orange, and black walls represent semiconducting metallic, and insulating tubes chiralities. ON-state $\left(\mathrm{V}_{\mathrm{g}}=-40 \mathrm{~V}\right)$ before (black) and after (red) covalent functionalization I-V curves of an electrically broken down DWCNT TFT within the (b) first, (e) second, and (h) third regime. OFF-state ( $\mathrm{Vg}=40 \mathrm{~V}) \mathrm{I}-\mathrm{V}$ curves for DWCNT conduction channels within the first (c), second (f), and third regime (i) before covalent functionalization. 


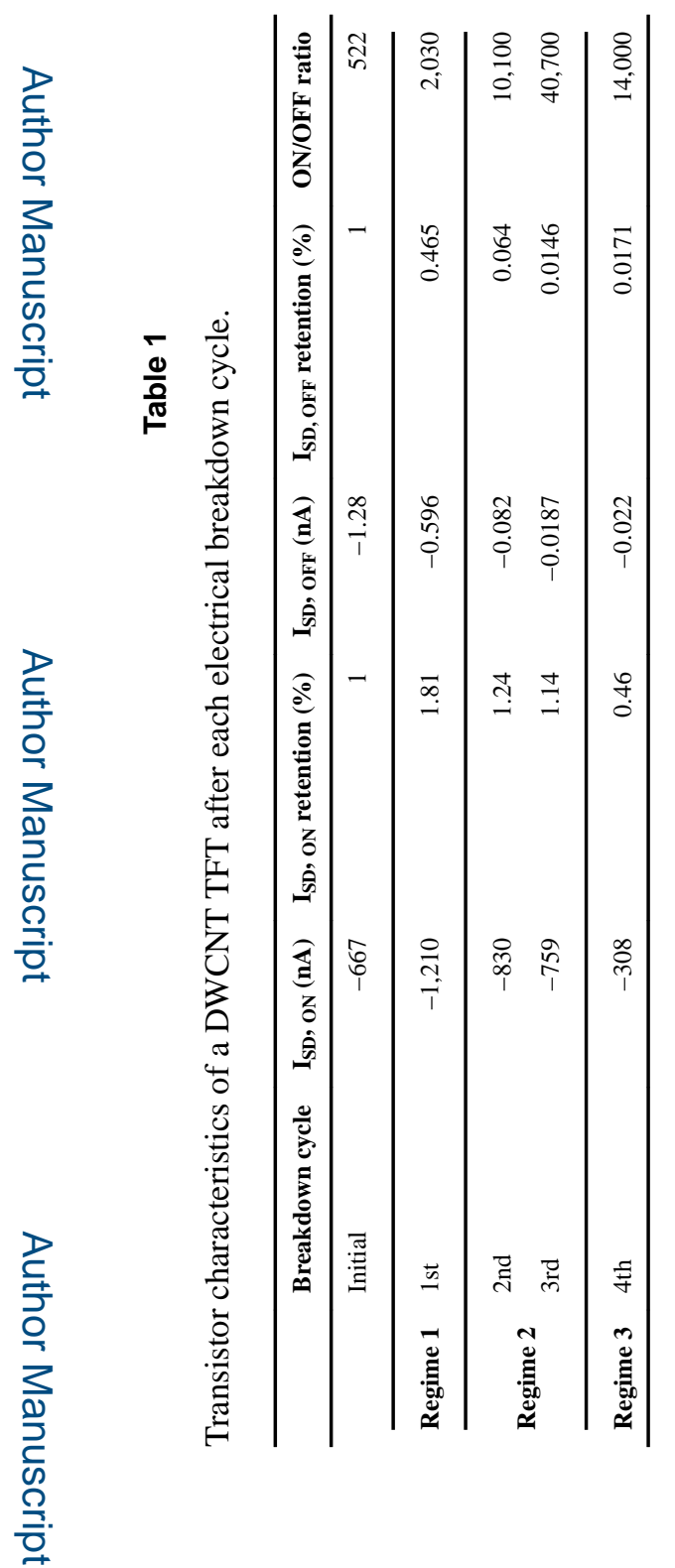

Small. Author manuscript; available in PMC 2016 July 06. 